\title{
Movement Pattern Recognition of Medaka for an Insecticide: A Comparison of Decision Tree and Neural Network
}

\author{
Yountae Kim, Daehoon Park, and Sungshin Kim \\ School of Electrical and Computer Eng., Pusan National University
}

\begin{abstract}
Behavioral sequences of the medaka (Oryzias latipes) were continuously investigated through an automatic image recognition system in response to medaka treated with the insecticide and medaka not treated with the insecticide, diazinon $(0.1 \mathrm{mg} / \mathrm{l})$ during a 1 hour period. The observation of behavior through the movement tracking program showed many patterns of the medaka. After much observation, behavioral patterns were divided into four basic patterns: active-smooth, active-shaking, inactive-smooth, and inactive-shaking. The "smooth" and "shaking" patterns were shown as normal movement behavior. However, the "shaking" pattern was more frequently observed than the "smooth" pattern in medaka specimens that were treated with insecticide. Each pattern was classified using classification methods after the feature choice. It provides a natural way to incorporate prior knowledge from human experts in fish behavior and contains the information in a logical expression tree. The main focus of this study was to determine whether the decision tree could be useful for interpreting and classifying behavior patterns of the medaka.
\end{abstract}

Key words: Pattern Recognition, Behavioral Patterns, Medaka, Decision Tree, Neural Network

\section{Introduction}

Ecological data are very complex, unbalanced, and contain missing values. Relationships among variables may be strongly nonlinear and involve high-order interactions. The commonly used exploratory and statistical modeling techniques often fail to find meaningful ecological patterns from data [1-3]. The behavioral or ecological monitoring of water quality is important regarding bio-monitoring and risk assessment [4], [5]. An adaptive computational method was utilized to analyze behavioral data in this study. There are many methods in the field of classification methods. In this paper, we classified data by the decision tree and neural network learning and compared the performance of them. The decision tree is a modern statistical technique that is ideally suited for both exploring and modeling data. It is constructed by repeatedly splitting the data, and defined by a simple rule based on a single explanatory variable. And neural network is also a good technique that is used for classification. It is very easy and powerful method.

In recent years, research on the effects of sub-lethal levels of toxic substances has been rapidly accumulating for various taxa, including crustaceans [6], snails [7], fish [8], [9], and insects [10]. However, these studies are mostly based on observation of single or combinations of single behaviors with qualitative descriptions. Not much quantitative research has been conducted on behavioral changes in spatial and temporal

Manuscript received Mar. 8, 2007; revised Mar. 14, 2007.

This work was supported by the Second-stage of the Brain Korea 21 project in 2007. domains in response to treatments of toxic chemicals. The observation of the movement tracks of small sized animals has been separately initiated in the field of search behavior in chemical ecology [11] and computational behavior [12], [13]. In regard to searching behavior, the servometor and other tools were used for investigating the continuous movement tracks of insects, including cockroaches, in characterizing the effects of wind [14], pheromone [15], [16], relative humidity [17], and sucrose feeding [18]. In regard to the computational aspects [12], Alt modeled the movement of the organism, such as the circling path of gametes or the meander search by isopods, and Scharstein [19] revealed a complex directional autocorrelation function with monotonic decay and discontinuity at the origin. Tourtellot et al. [13] analyzed the movement length and turn definition in the analysis of the orientation data of cockroaches. Johnson et al. and Weins et al. attempted to quantify insect movements and suggested the fractal dimensions of pathway configurations [20], [21]. Recently, studies on rats were conducted in dynamic perspectives and statistical discrimination of motion in exploration behavior [22], [23].

These computational methods convey useful mathematical information regarding similarities present in the data of the movement tracks; for instance, correlation coefficients or fractal dimensions. Using these methods, however, the parameters are obtained through mathematical transformations of the movement data, and information is in a generally highly condensed state. These methods are usually not interpretable for uniquely and directly characterizing the actual shape of the movement tracks. 
In this paper, we utilized two kinds of classification methods for the classification of response behaviors and attempted to explain the shapes of the movement tracks through feature extraction in response to sub-lethal treatments of an insecticide. The decision tree and neural the network learning are a widely used technique for data classification and prediction. One of their advantages is that rules, which are easy to understand, can be induced. Realizing that there is a limit to observing with the naked eye, computational methods were used to conduct our research more effectively. First, statistical analysis in total moving distance, average speed, and sectional domination was conducted as a feature extraction. Furthermore, we devised a new analysis method for pattern isolation based on a decision tree and neural network learning to differentiate the patterns we thought distinctive. This research can help the biosensor field in detecting defects in fish, or in finding out chemical toxicants that exist in the water by observing specific behavior patterns of fish.

\section{Experiments for Data Acquisition}

\subsection{Rearing of Experimental Fishes}

The specimens of medaka (Oryzias latipes) used in our experiment were obtained from the Toxicology Research Center, Korea Research Institute of Chemical Technology (KRICT; Taejon, Korea). Only the specimens six to twelve months old were used. The medaka is about $4 \mathrm{~cm}$ in length and lives for about 1-2 years. Because it is an easy species to rear and reproduce, it is used widely in the research of genetics or as a testing material in the detection of water pollution.

\subsection{Experimental Conditions}

A day before experimentation, the medaka was put into the observation tank and was given approximately twelve hours to adjust. The specimens were kept in a temperature of $25^{\circ} \mathrm{C}$ and were given a sufficient amount of oxygen during these twelve hours prior to the experiment. The specimens used were male and about $4 \mathrm{~cm}$ long. In order to achieve image processing and pattern recognition effectively, a stable condition was maintained in the monitoring system. Disturbances to observation tanks and changes in experimental conditions were minimized. Aeration, water exchange and food were not provided to the specimens during the observation period and the light regime was kept consistent.

\subsection{Observation System}

The observation system used in the experiment is comprised of an observation tank $\left(80 \times 30 \times 10 \mathrm{~cm}^{3}\right.$, glass $)$, a water circulator with a temperature controller (Jisico ${ }^{\mathrm{TM}}$ ), a temperature data logger (Hobo), a CCD camera (HiTron, DSP HDC4N04SKR), a video overlay board (Video Overlay Board: Sigma $\Pi{ }^{\mathrm{TM}}$, SigmaCom, Korea), and an image recognition system. The aquarium used was based on the experiments on fish behavior in KRICT. The observed aquarium size was $40 \mathrm{~cm} \times 20 \mathrm{~cm} \times 10 \mathrm{~cm}$. Diazinon (DongYang Chemical; O,Odiethyl O-2-isopropyl-4-methyl-6-pyrimidyl thiophosphate, $93.9 \%$ ) dissolved in dimethylsulfoxide (DMSO; $10 \mathrm{mg} / \mathrm{l}$ ), was introduced at the concentration of $0.1 \mathrm{mg} / \mathrm{L}$ directly into an aquarium in which a 6-12 month old individual adult medaka specimen resided. During the period of observation, individual medaka specimens were placed in a glass aquarium. The analog data captured by the camera set in front of the aquarium were digitized by using the video overlay board every 0.25 seconds and were sent to the image recognition system to locate the target in spatial time domains. The spatial position of the medaka was recorded in two-dimensional $\mathrm{x}, \mathrm{y}$ coordinate values. After giving the experimenting specimen approximately twelve hours to adjust to the observation aquarium, the experiment was started. The experiment was started at about 8:00 8:30 AM every day. Each data from a movement pattern had an interval of one minute [24], [25].

\section{Feature Extraction Process}

\subsection{Images of Movement Behavir of Medaka}

In this paper, the movement patterns of the medaka were classified into shaking and smooth patterns as shown respectively in Fig. 1 and 2. When a medaka was affected by diazinon $(0.1 \mathrm{mg} / \mathrm{l})$, the treated specimen was generally less active, and the movement behavior was shaky and interspersed with irregular, repetitive back-and-forth movements. Response behaviors were frequently vertical as can be observed in Fig. 1, and the degree of shaking was usually higher during the upward climb. Fig. 2 shows generally not be treated species. The behavior of the medaka in a minute period of time was used to classify them into 5 patterns: active-smooth, activeshaking, inactive-smooth, inactive-shaking, and not determined in each case. "Not determined" means that a pattern was not classified into any one of these four categories. By the observation of an expert in fish behavior to initiate pattern isolation, the features were observed and the following three feature variables could be defined: high-speed ratio, FFT (Fast Fourier transformation) to angle transition, and projection to $x$ and $y$-axes. Fig. 3 shows the schematic diagram during one minute of the movement analysis for the process of extracting three distinctive characteristics from the data acquired and classifying 5 patterns based on this information. It is possible that some patterns may not have been classified for medaka treated with sub-lethal chemicals. However, in these cases, further analysis and observation can add new patterns and update the unsupervised classification method. 

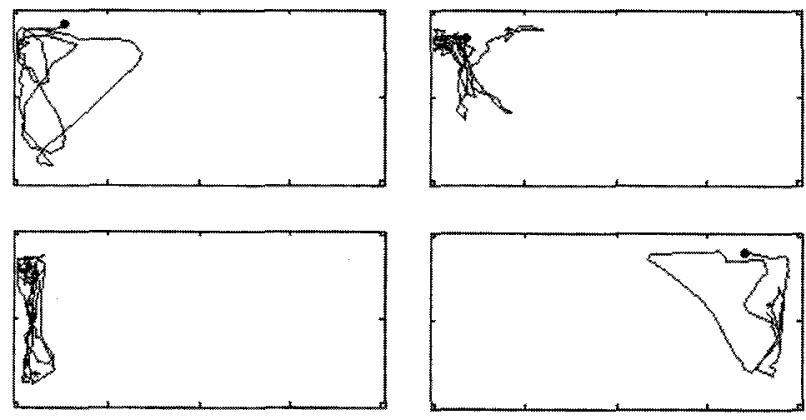

Fig. 1. Example of shaking patterns during a one-minute interval $(\bullet:$ start, $*$ : end $)$
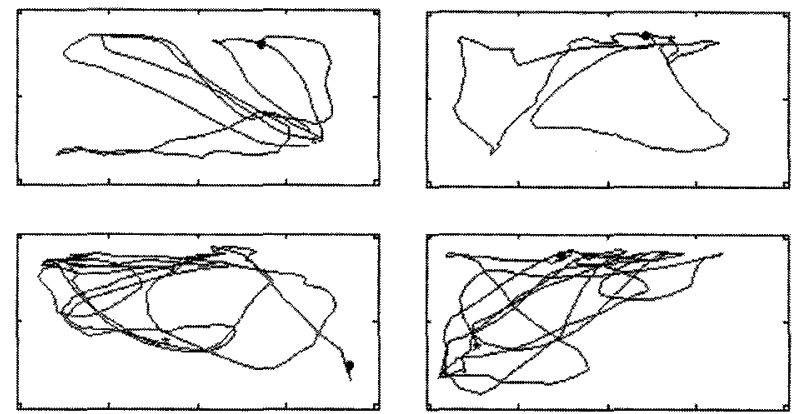

Fig. 2. Example of smooth patterns in a one-minute interval $(\because$ start, *: end $)$

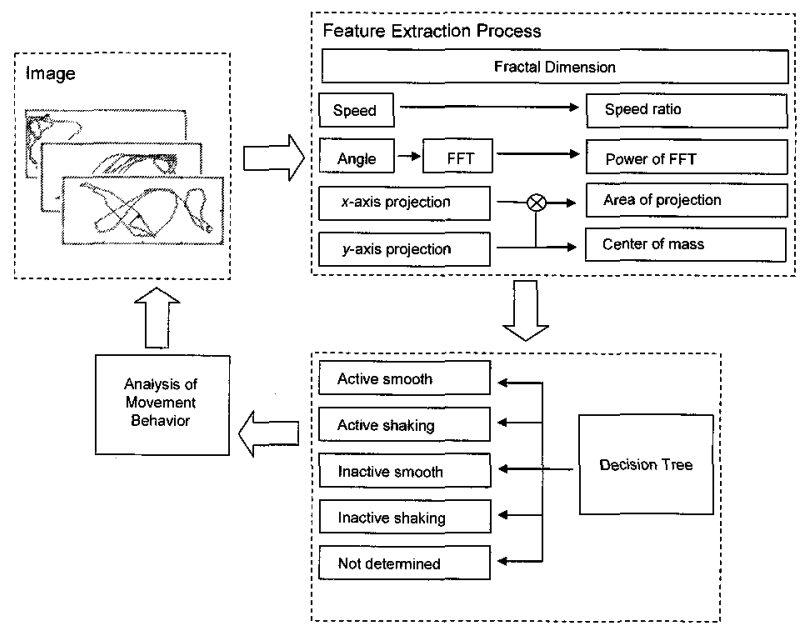

Fig. 3. Schematic diagram for automatic pattern isolation

\subsection{Feature Extraction from Images}

\subsubsection{Speed Ratio}

In order to know the activeness of a medaka, speed information was used to define high-speed ratio. The speed of the medaka shows whether the pattern is an active movement or inactive movement. The formula for speed is as the following:

$$
S=\sqrt{\left(x_{n+1}-x_{n}\right)^{2}+\left(y_{n+1}-y_{n}\right)^{2}} \quad n=1,2,3, \cdots
$$

Here, $x_{n}$ and $y_{n}$ are the position values of the medaka in a sampled time. The ratio of patterns that exceeded the calculated average speed of the overall 7 data sets, $21 \mathrm{~mm} / \mathrm{sec}$ and the total number of patterns was used as the first feature variable. Highspeed ratio is calculated using the following equation. A2 represents the average speed of the overall 7 data sets in equation (2).

$$
S_{\text {ratio }}=\frac{\text { Number of samples above A2 }}{\text { Number of samples in one minute }} \times 100(\%)
$$

\subsubsection{FFT to angle transition}

The change of direction in the movement track was observed to consider movements of medaka. The change of direction is represented as an angle transition to classify the movement. Angle transition between two sampled times denoted as $H$ is calculated in the following equation. Here $x_{n}$ and $y_{n}$ show the coordinate value for the $x$ and $y$ axes.

$$
H=\arctan \left(\frac{y_{n+1}-y_{n}}{x_{n+1}-x_{n}}\right), \quad n=1,2, \cdots
$$

Fourier transformation is used to transform signals in the time domain to signals in the frequency domain [26]. We apply the Fast Fourier Transform (FFT) to the signal of angle transition in order to calculate energy. The FFT for a given discrete signal $x[n]$ is calculated using the following equation:

$$
X[k]=\sum_{n=0}^{N-1} x[n] \cdot e^{-j(2 \pi k n / N)}, \quad k=0,1, \cdots, N-1 .
$$

After applying the FFT to angle transition, the power of FFT (PF) is calculated using the following equation for the amplitudes above a median.

$$
P=\sqrt{\sum_{i=1}^{k} x_{i}^{2}}
$$

Here, $x_{i}$ is the amplitude above a median. We use all sets to find the median in experiments. We are used to FFT power because of the calculation in qualified angle transition. The PF is employed as the second feature variable for pattern isolation.

\subsubsection{Projection}

Projection is a method of showing a shape in a twodimensional graph into a shape in a one-dimensional graph. In this paper, the method of projection was used to observe and understand the movement route of the medaka in a twodimensional space. The projection to the $x$-axis and the 
projection of the $y$-axis were calculated and then multiplied to figure out the area of the movement track of the medaka. The calculated area tells whether the medaka moved broadly all over the tank or in a restricted area of the tank. The area calculated was used as the third variable to classify patterns.

\subsubsection{Fractal Dimension}

In the fractal dimension, the spherical space, a cube or any other solid bodies are 3 dimensions and a perfect square or triangle is 2 dimensions a straight line or a curved line is 1 dimension the point is 0 dimension. The fractal dimension represented a value between 1 dimension and 2 dimensions depend on the bow degree. We analyzed the movement behavior of medaka using the fractal dimension as the characteristic variable. We defined the dimension about the random shape in the fractal geometry as the equation as follows.

$$
N=r^{D_{f}}
$$

Where, $N$ is a subpart number in each step, $r$ is a scaling factor. Log scale at both sides of the equation (6), fractal dimension $D_{f}$ is as follows.

$$
D_{f}=\frac{\ln N}{\ln r}
$$

\subsubsection{Center of Mass}

Another variable to represent the movement behavior of medaka is the center of mass. Because of the characteristics of the medaka, medaka usually goes up to the surface of the water in order to breathe and to eat foods. So in this paper, to record and classify the trace when medaka stayed around the surface of the water we used the center of mass. The equation of the center of mass is as follows. The center of mass used in this paper, applied only the value about $y$-axis to know the vertical position of the medaka.

\section{Pattern Recognition Results}

\subsection{Decision Tree Result}

We analyzed movement tracks of the medaka using MTP. The decision tree is employed and programmed to express the classification in the form of a tree and as a set of $I F-T H E N$ rules. In order to classify the patterns into active smooth, active shaking, inactive smooth and inactive shaking divided by the experts in fish behavior, the following features were used: high speed ratio (HSR), power of FFT (PF), and area of projection product (APP). These 3 features were used as input variables for the decision tree. The training data for the decision tree consisted of 30 data in each pattern: active smooth, active shaking, inactive smooth, inactive shaking, and not determined.
We continue splitting nodes in successive layers until the error on the validation data is minimized. Cross validation is a model evaluation method that is better than residuals. The problem with residual evaluations is that they do not give an indication of how well the learner will do when it is asked to make new predictions for data it has not already seen. One way to overcome this problem is to not use the entire data set when training a learner. Some of the data is removed before training begins. Then when training is done, the data that was removed can be used to test the performance of the learned model. We used a 10 -fold cross validation for model evaluation. The data set of the 10 -fold cross validation is divided into 10 subsets, and the holdout method is repeated 10 times. Result of this method is shown in Fig. 4. The principal alternative approach to stopped splitting is pruning. Fig. 5 shows the decision tree applied to evaluated pruning. This benefit of this logic is that the reduced rule set may give improved interpretation. At last, we tested decision tree model with 50 data that is consist of 10 data from 5 patterns. Table 1 shows the result we tested decision tree model.

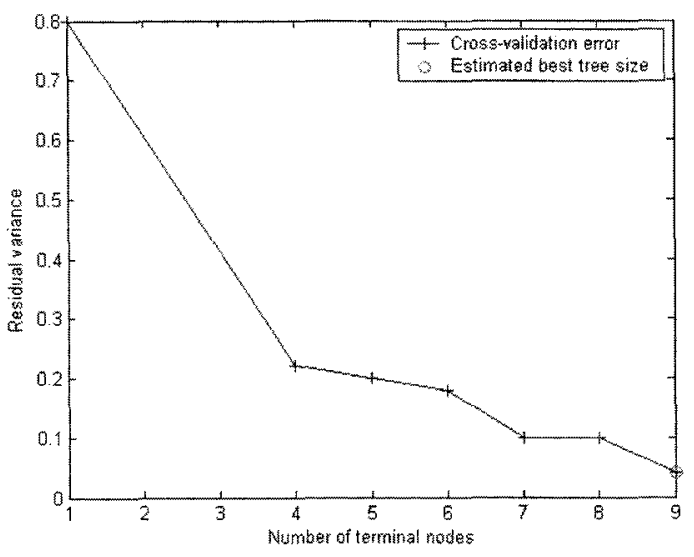

Fig. 4. Result of cross-validation (hold-out method)

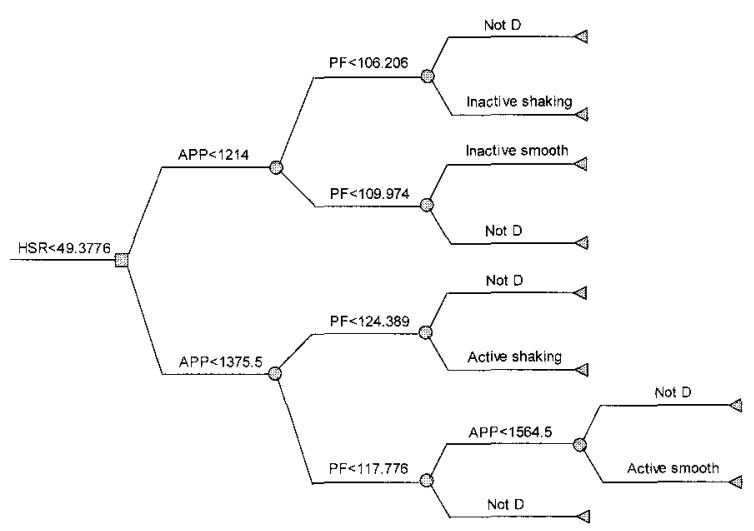

Fig. 5. The decision logic for pattern classification generated by decision tree applied to pruning. (HSR: high-speed ratio, APP: area of projection product, PF: power of FFT) 
Table 1. Recognition rate of decision tree model.

\begin{tabular}{|c|c|c|c|c|c|c|}
\hline & $\begin{array}{c}\text { Active } \\
\text { shaking }\end{array}$ & $\begin{array}{c}\text { Aetive } \\
\text { smooth }\end{array}$ & $\begin{array}{c}\text { Inactive } \\
\text { shaking }\end{array}$ & $\begin{array}{l}\text { Inactive } \\
\text { smooth }\end{array}$ & deternined & Total \\
\hline $\begin{array}{c}\text { Selected by } \\
\text { expert }\end{array}$ & 10 & 10 & 10 & 10 & 10 & 50 \\
\hline $\begin{array}{c}\text { Tested by } \\
\text { model }\end{array}$ & 10 & 9 & 9 & 9 & 8 & 45 \\
\hline
\end{tabular}

\subsection{Neural Network Result}

We used same data that is used to construct a decision tree model to verify and learn neural network. In other words, we extracted thirty data from each chosen patterns with the advice from experts in fish behavior. We chose five numerical values for the distinctive characteristics as input values and the pattern as an output value. Preprocessing is essential in neural network learning. The first reason why we preprocess the learning data is that the importance of each input data is same and to protect the logistical transition function from saturation quickly. And the second reason is that output or goal data are within the limit of the range that transition function can get. We performed the linear transform about the transform of input data, and the equation is as follows

$$
Y=\frac{0.8}{M-m}(X-m)+0.1
$$

Here, $M$ is maximum input value, $m$ is the minimum input value, $X$ is input value, and $Y$ is transition value. Transition data value is in 0.1 to 0.9 . This transition function can be used to transform the goal value. When logistical transition function is used, the output always has to be transformed between 0.1 and 0.9 . In order to pattern recognition we organized neural network model. Neural network is made up of three layers, which is consist of input layer, hidden layer, output layer. We used 5 nodes for input layer, 4 nodes for hidden layer, and 1 node for output layer. We classified 5 patterns first. 5 patterns are consist of 4 patterns classified by experts in fish behavior and 'not determined' that is not belonged 4 patterns by experts. In order to neural network learning, we chose 5 characteristics from 5 patterns as inputs. We performed learning with 150 data that is consists of 30 data from each pattern. We have 2000 epoch to learn enough.

We tested neural network model with 50 data that is consist of 10 data extracted from every 5 behavior patterns. We organized neural network using MATLAB tool box and Simulink. Fig. 6 shows neural network structure for movement behavior estimation. Fig. 7 shows the result we tested neural network with 10 data from every behavior patterns. Table 2 shows the recognition rates of neural network model. Results are similar to decision making tree model at Active shaking, active smooth, inactive shaking, inactive smooth pattern. But recognition rate at not determined pattern is a little low value.
In this result, we chose the decision making tree model to classify the training data.

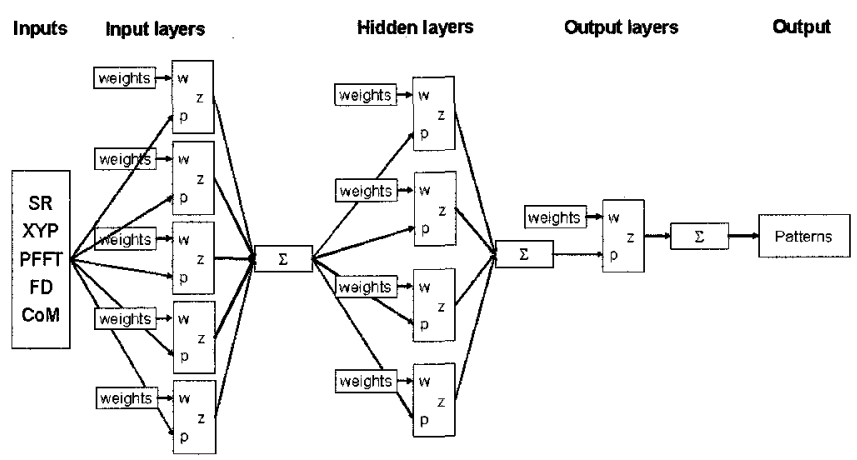

Fig.6. Neural network structure for movement behavior estimation

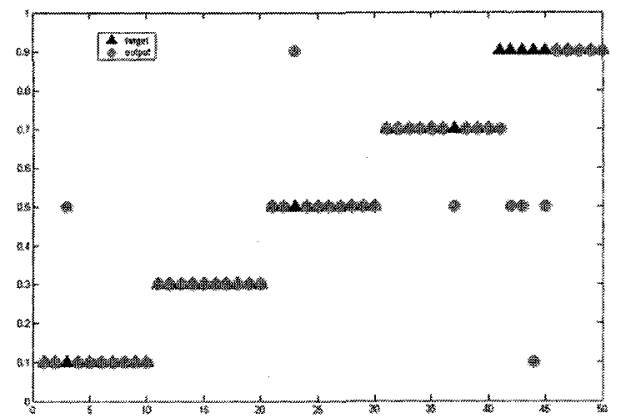

Fig.7. Results of the neural network.

Table 2. Recognition rates of neural network model.

\begin{tabular}{|c|c|c|c|c|c|c|}
\hline & $\begin{array}{c}\text { Active } \\
\text { haking }\end{array}$ & $\begin{array}{c}\text { Active } \\
\text { smooth }\end{array}$ & $\begin{array}{c}\text { nactive } \\
\text { shaking }\end{array}$ & $\begin{array}{c}\text { nactive } \\
\text { smooth }\end{array}$ & determined & Total \\
\hline $\begin{array}{c}\text { Selected } \\
\text { by expert }\end{array}$ & 10 & 10 & 10 & 10 & 10 & 50 \\
\hline $\begin{array}{c}\text { Tested by } \\
\text { model }\end{array}$ & 9 & 10 & 9 & 9 & 5 & 42 \\
\hline
\end{tabular}

\section{Behavior Analysis and Discussion}

\subsection{Analysis of Movement Behavior}

The decision tree was applied into the movement tracks of the medaka at real-time to classify patterns. We developed models based on the classification and regression tree (CART) in order to classify and recognize movement tracks of the medaka for an insecticide. Matlab7.1 was used in order to create the program. Results were calculated for the decision logic for 60 minutes. The specimens used in the experiment were 10 medakas treated with insecticide and 10 medakas not treated with insecticide. The recognition is calculated by 5 patterns that includes "not determined." "Smooth" means that "active smooth" patterns and "inactive smooth" patterns appeared in the decision tree logic. "Shaking" means that "active shaking" patterns and "inactive shaking" patterns appeared in the decision tree logic. "Not determined" means that neither "smooth" nor "shaking" appeared in the decision 
tree logic. Fig. 8 shows the ratio of "smooth", "shaking" and "not determined" patterns in specimens without the insecticide. "Smooth" is the sum of active smooth and inactive smooth patterns. "Shaking" is the sum of active shaking and inactive shaking. "Not" is the sum of patterns that are not inactive shaking, not inactive smooth, not active shaking, and not active smooth shown in Fig. 5. Among the 10 data sets, the recognition rate of specified patterns in smooth and shaking is in a range of $48 \sim 77 \%$ while the rate of average is $62.1 \%$. Most specimens showed more smooth patterns detected by the decision tree logic. Fig. 9 shows the ratio of "smooth", "shaking" and "not determined" patterns in medaka that were treated with medaka. Among the 10 data sets, the recognition rate of specified patterns in smooth and shaking is $23 \sim 57 \%$ and the recognition rate of average is $40.5 \%$. The shaking pattern has a higher observed rate than the smooth pattern regarding to the medaka treated with insecticide The lower recognition rate is observed in medaka that were treated with the insecticide compared to the medaka that were not treated with the insecticide. Unlike the data sets of specimens without the insecticide, the data sets A', B', C' which exceeded a $50 \%$ recognition rate showed many shaking patterns. However, the data sets of less than $50 \%$ showed a similar rate of smooth and shaking patterns.

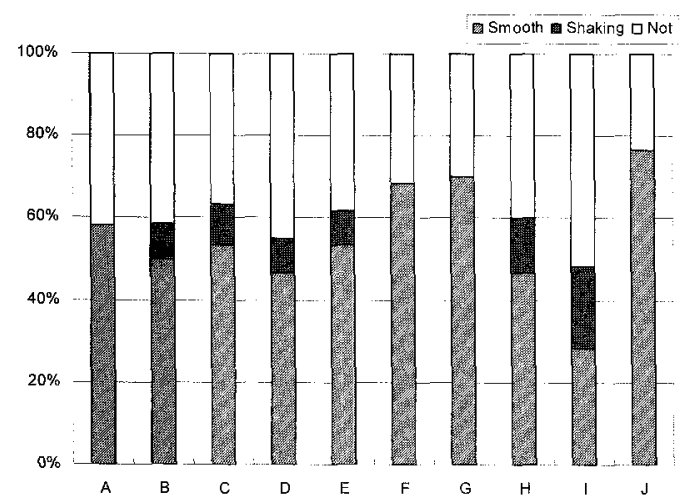

Fig. 8. Recognition rate for each pattern in set of medaka without the insecticide

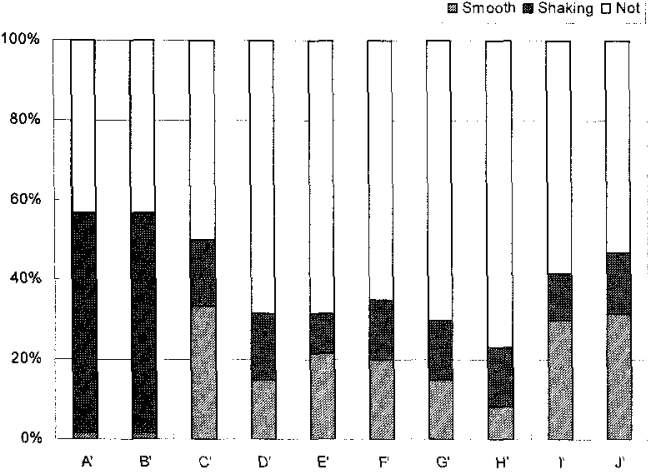

Fig. 9. Recognition rate for each pattern in set of medaka with insecticide

\subsection{Discussion}

This study demonstrated that behavioral differences of animals in response to an insecticide could be detected by a decision tree using 5 features of behavior. One difficulty of conducting this type of monitoring study is the necessity of handling a large amount of data. In this study, the data were produced in every 0.25 second interval continuously for each individual measurement period. This produced a gigantic amount of data. The automatic pattern recognition system solved this time-consuming problem in detecting response behaviors. Besides time consumption in recognition, objectivity in judgments for classification has been another problem for manual recording. The application of machine intelligence to behavioral data has the advantage of classifying the movement patterns on a more objective basis. In this regard, the pattern recognition by a decision tree was demonstrated as an alternative to detecting the movement tracks of animals. These points will help in the analysis of behavior patterns in not only temperature elevation but also in different types of chemicals. Another problem that arouses from this experiment is that biological specimens such as the medaka show too many different types of movement patterns. This makes selecting certain characteristics for a pattern difficult. This is why so many artificial systems such as neural networks and fuzzy are being used [27-30]. However, although neural networks are sufficiently able to differentiate patterns, it is impossible to interpret exactly how much a certain pattern the specimen shows.

Results revealed that after differentiating smooth and shaking patterns through classification methods, insecticide treatment caused the shaking ratio to increase. This can be seen as a pattern that appears in response to sub-lethal treatments of an insecticide, and is a process of adaptation. Smooth patterns show less angle change than shaking patterns and can be seen as a pattern without insecticide treatment, and it can be said that it appears the most frequently. Through this research, classification methods were devised using 4 characteristic patterns and 'not determined' for the patterns that could not be defined, based on the knowledge of experts. The used classification methods were able to differentiate the 4 patterns based on the observation of the five variables. And we could find the decision tree is better than neural network in this situation. However, more research must be done in order to define the patterns that were 'not determined.' Also, in order to better observe the many movement patterns of the medaka, more data sets should be examined and studied. Biologically, results showed that variables such as smooth ratio vs. shaking ratio distinguished before and after insecticide treatment in Fig. 8 and 9 . It can be inferred from these results that the activity did increase as the treatment began to rise. Although this is a 
short period of time it may be seen as a case of fast acclimation to the insecticide by the medaka.

\section{Conclusions}

The complex movement data were used to be classified using the decision tree logic and neural network learning with 5 features that could represent the movement tracks of medaka: speed ratio, power of FFT, $x$ - and $y$-axes projection product, fractal dimension and center of mass. As new input data were given to classification methods, it was possible to recognize the change of pattern by examining the availability of insecticide. In these cases, a new analysis can be done to add new patterns and update. The results of the decision tree revealed that whether the medaka was affected by insecticide or not, it interpreted speed, angle, area of projection to $x$ - and $y$-axes, fractal dimension and center of mass using decision tree logic. If this is applied to more data sets, it is thought that more distinctive and accurate methods of differentiating the behavior patterns can be created. Also, this research in differentiating patterns may help in the field of research for the special characteristics of living organisms. This research can help the biosensor field in detecting defects in fish, or in finding out other chemical toxicants that exist in the water by observing specific behavior patterns of fish.

\section{References}

[1] L. Breiman, J. H. Friedman, R. A. Olshen, C.G. Stone, Classification and Regression Trees, Wadsworth International Group, Belmont, California. USA, 1984.

[2] B. D. Ripley, Pattern recognition and neural networks, Cambridge University Press, Cambridge, UK, 1996.

[3] O. D. Richard, E. H. Peter, G. S. David, Pattern Classification 2nd edn, Wiley Interscience, USA, 2001.

[4] H. Dutta, J. Marcelino, Ch. Richmonds, "Brain acetylcholinesterase activity and optomotor behavior in bluefills, Lepomis macrochirus exposed to different concentrations of diazinon," Arch. Intern. Physiol. Biochim. Biophys., Vol. 100, No. 5, pp. 331-334, 1993.

[5] A. D. Lemly, R. J. Smith, "A behavioral assay for assessing effects of pollutants of fish chemoreception," Ecotoxicology and Enviornmental Safety, Vol. 11, No. 2, pp. 210-218, 1986.

[6] S. D. Roast, J. Widdows, M. B. Jones, "Disruption of swimming in the hyperbenthic mysid Neomysis integer (Peracarida: Mysidaces) by the organophosphate pesticide chlorpyrifos," Aquatic Toxicology, Vol. 47, pp. 227-241, 2000.
[7] W. L. F. Ibrahim, P. Furu, A. M. Ibrahim, N. O. Christensen, "Effect of the orgaophosphorous insecticide, chlorpyrifos (Dursban), on growth, fecundity and mortality of Biomphalaria aexandrina and on the production of Schistosoma mansoni cercariae in the snail," Journal of Helminthology, Vol. 66, pp. 79-88, 1992.

[8] A. Moore, C. P. Waring, "Sublethal effects of the pesticide diazinon on olfactory function in mature male Atlantic salmon parr," Journal of Fish Biology, Vol. 48, pp. 758$775,1996$.

[9] M. A. Gray, K. L. Teather, C. D. Metcalfe, "Reproductive success and behavior of Japanese medaka (Oryzias latipes) exposed to 4-tera-octylphenol," Environmental Toxicology and Chemistry, Vol. 18, pp. 2587-2594, 1999.

[10] T. S. Chon, Y. S. Park, M. H. Ross, "Activity of German cockroach, Blattella germanica (L.) (Orthoptera : Blattellidae), at different microhabitats in semi-natural conditions when treated with sublethal doses of pesticides," Journal of Asia-Pacific Entomology, Vol. 1, pp. $77-83,1998$.

[11] W. J. Bell, "Searching behavior patterns in insects," Annual Review of Entomology, Vol. 35, pp. 447-467, 1990.

[12] W. Alt, G. Hoffman(Eds), Biological Motion. Lecture notes in Biomathematics 89, Springer-Verlag, Berlin, 1989.

[13] M. K. Tourtellot, R. D. Collins, W. J. Bell, "the problem of movelength and turn definition in analysis of orientation data," Journal of Theoretical Biology, Vol. 150, pp. 287-297, 1991.

[14] W. J. Bell, E. Kramer, "Search and anemotactic orientation of cockroach," Journal of Insect Physiology, Vol. 25, pp. 631-640, 1975.

[15] W. J. Bell, E. Kramer, "Sex pheromone-stimulated orientation of the American cockroach on a servosphere apparatus," Journal of Chemical Ecology, Vol. 6, pp. 287-295, 1980.

[16] W. J. Bell, R. T. Tobin, "Orientation to sex pheromone in the American cockroach: analysis of chemo-orientation mechanisms," Journal of Insect Physiology, Vol. 27, pp. 501-508, 1981.

[17] K. A. Sorensen, W. J. Bell, "Orientation responses of an isopod to temporal changes in relative humidity simulation of a "humid patch" in a "dry habitat"," Journal of Insect Physiology, Vol. 32, pp. 51-57, 1986.

[18] J. White, T. R. Tobin, W. J. Bell, "1984. Local search in the house fly Musca domestica after feeding on sucrose," Journal of Insect Physiology, Vol. 30, pp. 477-487, 1984.

[19]H. Scharstein, Paths of carabid beetle walking in the absence of orienting stimuli and the time structure of their motor output. In: Alt, W., Hoffmann, G. (Eds), Biological Motion. Lecture Notes in Biomathematics 89, SpringerVerlag, Berlin, pp. 269-277, 1989.

[20] A. R. Johnson, B. T. Milne, J. A. Wiens, "Diffusion in fractal landscapes: simulations and experimental studies of 
tenebrionid beetle movements," Ecology, Vol. 73, pp. 1968-1983, 1992.

[21] J. A. Weins, T. O. Crist, K. A. With, B. T. Milne, "Fractal patterns of insect movement in microlandscape mosaics," Ecology, Vol. 79, No. 2, pp. 663-666, 1995.

[22]O. Tchernichorski, Y. Benjamini, I. Golani, "The dynamics of long-term exploration in the rat Part I A phase-plane analysis of the relationship between location and velocity," Biological Cybernetics, Vol. 78, pp. 423432, 1998.

[23] O. Tchernichovski, Y. Benjamini, "The dynamics of longterm exploration in the rat Part II A phase-plane analysis of the relationship between location and velocity," Biological Cybernetics, Vol. 78, pp. 433-440, 1998.

[24] S. T. Lee, J. H. Kim, J. Y. Baek, M. W. Han, S. S. Kim, T. S. Chon, Pattern Analysis of Movement Behavior of Medaka (Oryzias latipes): A Decision Tree Approach. In: André Gagalowicz, Wilfried Philips (Eds), Computer Analysis of Images and Patterns. Lecture Notes in Computer Science, Springer-Verlag, Berlin, Vol. 3691, pp. 546-554, 2005.

[25]S. T. Lee, S. S. Kim, "Classification and Recognition of Movement Behavior of Animal based on Decision Tree," Journal of Fuzzy Logic and Intelligent Systems (Korean), Vol. 15, N o. 6, pp. 682-687, 2005.

[26] Erwin Kreyszig, Advanced Engineering Mathematics, 8th Ed, Wiley, 1999

[27] T. S. Chon, Y. S. Park, K. H. Moon, E. Y. Cha, "Patternizing communities by using an artificial neural network," Ecological Modeling, Vol. 90. pp. 69-78, 1996.

[28] I. S. Kwak, T. S. Chon, H. M. Kang, N. I. Chung, J. S. Kim, S. C. Koh, S. K. Lee, Y. S. Kim, "Pattern recognition of the movement tracks of medaka (Oryzias latipes) in response to sub-lethal treatments of an insecticide by using artificial neural networks," Environmental Pollution, Vol. 120, pp. 671-681, 2002.

[29]S. S. Kim, H. Bae, M. H. Lee, "Design and optimization of fuzzy controllers based on the operator's knowledge," Artificial Life and Robotics, Vol. 6, pp. 92-98, 2002.

[30] Y. K. Woo, H. Bae, S. S. Kim, K. B. Woo, "Intelligent Methods to Extract Knowledge from Process Data in the Industrial Applications," International Journal of Fuzzy Logic and Intelligent Systems, Vol. 3, No. 2, pp. 194-199, 2003.

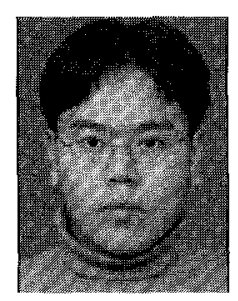

\section{Yountae Kim}

Yountae Kim received the B.S. and M.S. degrees in the School of Electronic, Electrical and Communication Engineering from Pusan National University, Busan, Korea, and is currently working in the doctor's course. His research interests are intelligent control, fuzzy logic control, and pattern recognition.

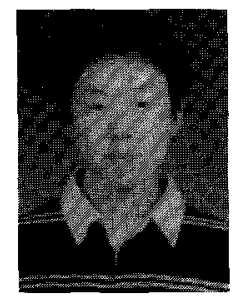

\section{Daehoon Park}

Daehoon Park received the B.S. degrees in the School of Electronic, Electrical and Communication Engineering from Pusan National University, Busan, Korea, and is currently working in the master's course. His research interests are intelligent control, fuzzy logic control, and neural networks

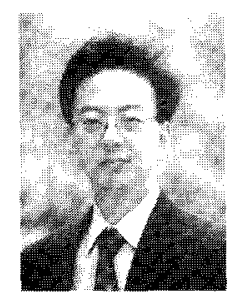

\section{Sungshin Kim}

Sungshin Kim (S'90-M'93) received the B.S. and M.S. degrees in electrical engineering from Yonsei University, Seoul, Korea, in 1984 and 1986, respectively, and the $\mathrm{Ph} . \mathrm{D}$. degree in electrical and computer engineering from Georgia Institute of Technology, Atlanta, in 1996. He is currently an Associate Professor in the School of Electronic and Electrical Engineering, Pusan National University, Busan, Korea. His research interests include intelligent systems, hierarchical learning structures, manufacturing systems, fuzzy logic control, and neural networks.

$\begin{array}{ll}\text { Phone } & :+82-51-510-2374 \\ \text { Fax } & :+82-51-513-0212 \\ \text { E-mail } & : \text { sskim@pusan.ac.kr }\end{array}$

